\title{
Lymphoproliferative diseases
}

In the last decade enormous progress has been achieved in the diagnosis and treatment of lymphoproliferative diseases. Molecular tools now allow a more precise disease classification and the allocation of individual patients to distinct prognostic subgroups. Treatment approaches more and more become "targeted therapies" containing new drugs as monoclonal antibodies and immunomodulatory or antiangiogenic substances.

In this issue of MEMO, we have set a focus on lymphoproliferative neoplasms. Distinguished experts review the standards in diagnosis and care of common lymphomas and also some rare neoplasms that often cause uncertainties about their management, e.g. primary CNS lymphomas and peripheral T-cell lymphomas are outlined.

Dr. Willenbacher is presenting an instructional case emphasizing the need of a fastidious and tenacious search for the bleeding source in anaemia due to iron deficiency in order not to overlook a malignant disease.

My sincere thanks to the authors for keeping their manuscripts tight although covering all major aspects of the management of the particular disease.

Conflict of interest

The authors declare that there is no conflict of interest.

Eberhard Gunsilius (Section Editor Haematology) 